\title{
Axial spondyloarthritis may protect against poor outcomes in COVID-19: propensity score matched analysis of 9766 patients from a nationwide multi-centric research network
}

\author{
Rahul Raiker $^{1}$ (D) Haig Pakhchanian ${ }^{2}$ (D) $\cdot$ Chengappa Kavadichanda ${ }^{3} \cdot$ Latika Gupta $^{4}$ (D) Sinan $\operatorname{Kardes}^{5}$ (D) \\ Sakir Ahmed ${ }^{6}$ D
}

Received: 17 June 2021 / Revised: 12 September 2021 / Accepted: 31 October 2021 / Published online: 27 November 2021

(C) International League of Associations for Rheumatology (ILAR) 2021

\begin{abstract}
Introduction The outcomes of COVID-19 in patients with axial spondyloarthritis (ax-SpA) have not been explored in detail. Tumour necrosis factor inhibitors (TNFi) are commonly used for ax-SpA patients, and how they influence outcomes may have implications on COVID-19 management.

Methods A nationwide multi-centric research network was queried for patients with ax-SpA, including ankylosing spondylitis (AS) and non-radiographic SpA (nr-SpA) who had developed COVID-19. An equal number of propensity score(PS) matched controls were extracted from the database amongst patients with COVID-19 who did not have any inflammatory arthritis. Outcomes included mortality and others including hospitalization, intensive care unit, ventilation, acute kidney injury (AKI), renal replacement therapy, acute respiratory distress syndrome, cerebral infarction, venous thromboembolism (VTE), and sepsis.

Results We identified 9766 patients with ax-SpA (924 AS and 8842 nr-SpA) and 691,862 without SpA who had COVID-19. In the unmatched comparison, patients with ax-SpA had higher risk ratios (RR) for all outcomes. After matching for demographics and comorbidities, patients with ax-SpA had lower RR for mortality [RR: 0.707 (95\% CI: 0.598-0.836), $p<0.0001]$, severe COVID-19 [RR: 0.791 (0.69-0.906), $p=0.0007$ ], hospitalization [RR: 0.872 (0.826-0.921), $p<0.0001$ ], and AKI [RR: 0.902 (0.816-0.997), $p=0.044]$. Only the risk of VTE was higher in ax-SpA patients [RR: $1.219(1.037-1.433), p=0.016]$. Amongst the ax-SpA group, males had worse outcomes in 9 out of the 11 domains except for VTE and cerebral infarction, while blacks had worse outcomes in all except for mortality and the need for renal replacement therapy. AS had similar risk ratios for all outcomes compared with nr-SpA except hospitalization [RR: $1.457(1.03-2.06), p=0.0318]$. There was no difference in outcomes in patients who had received TNFi in the year previous to COVID-19 infection. Ax-SpA patients who had been prescribed non-steroidal anti-inflammatory drugs in the 3 months prior to COVID-19 had poorer outcomes. Conclusion In conclusion, COVID-19 outcomes were better in patients with ax-SpA as compared with PS matched controls except for increased risk for VTE. The use of TNFi is not associated with better or worse outcomes. These apparently protective effects observed need to be validated and explored further.
\end{abstract}

Sakir Ahmed

sakir005@gmail.com

Extended author information available on the last page of the article 


\section{Key Points}

- Patients with axial spondyloarthritis have lower mortality and morbidity during COVID-19 infections as compared with propensity score matched controls.

- Axial spondyloarthritis is associated with higher risks for venous thromboembolism during COVID-19.

- There is no difference in outcomes between ankylosing spondylitis and non-radiographic spondyloarthritis except in rates of hospitalization, which were higher in ankylosing spondylitis.

- Use of tumour necrosis factor inhibitors did not influence COVID19 outcomes.

Keywords Ankylosing spondylitis · COVID-19 · Multicentric cohort $\cdot$ Outcomes $\cdot$ Spondyloarthritis

\section{Introduction}

In the last 18 months, the COVID-19 pandemic has claimed over 3 million lives and has left no human unaffected. Though vaccination is helping to control the pandemic, new infections are still emerging. Different autoimmune diseases and immunosuppressants are expected to influence the outcomes of COVID-19. To date, most published analyses of the effects of various rheumatic and musculoskeletal diseases (RMD) on COVID-19 outcomes have utilized heterogeneous groups of patients $[1,2]$. Overall, inflammatory rheumatic diseases were expected to have higher risks for infections and more severe disease. In-depth analyses of published case series have revealed this to be not true [3]. Having a connective tissue disease like lupus, but not inflammatory arthritis, may be linked to more severe COVID-19 [4, 5]. Thus, with growing numbers of COVID-19 available worldwide, there is a need to study the effect of individual diseases on COVID19 outcomes. If any disease has protective outcomes, it can be due to the medications used for that disease or due to some intrinsic protection due to the disease itself. One such disease is axial spondyloarthritis, ax-SpA. The outcomes of COVID-19 in spondyloarthritis (SpA) are limited to case reports. Moreover, the literature focusing on outcomes of COVID-19 in ax-SpA patients exclusively is sparse [6,7].

Tumour necrosis factor inhibitors (TNFi) are commonly used in the treatment of ax-SpA. Thus, exploring outcomes in $\mathrm{SpA}$ would also enable us to study the possible associations of TNFi use with COVID-19 outcomes. In the fight against COVID-19, a major thrust has been on the repurposing of existing drugs [8]. The success of steroids in reducing mortality in the RECOVERY trial has provided support for the use of immunosuppressants and anti-inflammatory agents [9]. Early in the pandemic, the use of TNFi was suggested for severe COVID-19 based on its biological nature [10]. Till date there is no evidence of any adverse effects of TNFi during COVID-19. There is some epidemiological data from psoriasis and inflammatory bowel disease cohorts to support a better outcome in patients using TNFi [11]. At least 3 to 4 randomized controlled trials exploring the role of TNFi in severe COVID-19 are expected to be completed in the latter part of the year $2021[11,12]$.

The multi-centric research network TriNetX compiles electronic information from multiple centres across the USA, with minor contributions from other countries. It includes diagnoses, therapies used, infections like COVID-19, and clinically meaningful outcomes. Previously, the multi-centric health network, TriNetX, had been used to find out the outcomes of 8540 patients with RMDs [2]. Thus, we took the opportunity provided by TriNetX to explore outcomes of COVID-19 in patients with ax-SpA and also to analyse the effects of TNFi.

\section{Methods}

The study was designed along the lines of previous publications $[2,13-15]$ as a retrospective cohort derived from the TriNetX network. Ankylosing spondylitis (AS) and non-radiographic SpA (nr-SpA) who had COVID-19 were included, and one-to-one matched controls were selected using propensity score (PS).

The TriNetX database contains anonymized data. It was queried based on either the International Classification of Diseases tenth revision (ICD-10), Current Procedural Terminology (CPT) codes, or Logical Observation Identifiers Names and Codes (LOINC). These extracted data included demographics, diagnoses, drugs, and clinical outcomes.

\section{Patient selection}

The SpA with COVID-19 cohort included all patients who were 18 years of age or older, had a pre-existing diagnosis of ax-SpA (AS or nr-SpA), and were diagnosed with COVID-19 anytime from January 20, 2020, to April 30, 2021. ICD-10 codes were used for the diagnosis of ankylosing spondylitis (M45.x) or axial spondyloarthritis (M46.1, M46.8, M46.9). Diagnosis of COVID-19 was based on ICD-10 codes (U07.1, U07.2, J12.81, B34.2, B97.21, B97.29) augmented by polymerase chain reaction (PCR) for SARS-CoV-2 results when available. The control cohort was extracted from all the other COVID-19 patients excluding known ankylosing spondylitis or axial spondyloarthritis (M45.x, M46.x) or any other form of inflammatory arthritis (M05.x, M06.x, L40.53). 


\section{Data extracted}

Demographic characteristics (age, sex, race, body mass index), presence of comorbidities (hypertension [ICD10 code: I10], chronic lower lung disease [ICD10 codes: J40-J47], diabetes mellitus [ICD10 codes: E08-E13], ischemic heart disease [ICD10 codes: I20-I25], chronic kidney disease [ICD10 code: N18], heart failure [ICD10 code: I50], cerebrovascular disease [ICD10 codes: I60-I69], nicotine dependence [ICD10 code: F17], and alcohol-related disorders [ICD10 code: F10]), and medication used preceding the diagnosis of COVID-19 were extracted. TNFi included were etanercept, adalimumab, infliximab, certolizumab, and golimumab. Also, the use of non-TNFi biologicals like interleukin 17 inhibitors (namely secukinumab, ixekizumab) were included.

\section{Outcomes}

COVID-19 clinical outcomes included mortality, hospitalization, intensive care unit (ICU) admission, mechanical ventilation, acute kidney injury, renal replacement therapy (RRT) including haemodialysis, acute respiratory distress syndrome (ARDS), cerebral infarction, venous thromboembolism (VTE), and sepsis. Severe COVID-19 was defined in this study as the composite of mechanical ventilation and mortality. All these outcomes were assessed within 30 days from the date when the diagnosis of COVID-19 had been made.

\section{Statistical analysis}

A one-to-one PS-based matching was carried out to identify a control group. The demographic and comorbidity variables mentioned above were used as factors or covariates in the PS matching that utilized a greedy nearest-neighbour algorithm with a calliper of 0.1 pooled standard deviations. Risk ratios (with 95\% confidence intervals) were calculated for the outcomes mentioned above, comparing with both unmatched and matched controls. The detailed methodology is provided elsewhere [13, 15]. Two-sided tests of significance were used. Statistical significance was fixed at a $p$-value of less than 0.05 . For visualization of results, R statistical program (Ver 4.0.3) was used along with the ggplot2 package and its dependencies.

\section{Ethics statement}

Due to its status as a federated network providing de-identified data with aggregated counts and statistical summaries, the TriNetX has been granted a waiver by the Western Institutional Review Board.

\section{Results}

We identified 9766 patients with ax-SpA who had COVID19 infection. Of these, 924 were coded as having AS and the other 8842 as nr-SpA. The mean age was $60.3 \pm 15.3,66.2 \%$ were females, and the majority $(74.3 \%)$ were white. After

Table.1 Baseline characteristics of axial spondyloarthritis (ax-SpA) versus the rest (unmatched and matched)

\begin{tabular}{|c|c|c|c|c|c|c|}
\hline \multirow[t]{2}{*}{ Parameters } & & \multirow[t]{2}{*}{ Ax-SpA patients } & \multicolumn{2}{|c|}{ Unmatched non-ax-SpA } & \multicolumn{2}{|c|}{ Matched non-ax-SpA controls } \\
\hline & & & Data & $\begin{array}{l}\text { Standardized } \\
\text { difference }\end{array}$ & Data & $\begin{array}{l}\text { Standard- } \\
\text { ized differ- } \\
\text { ence }\end{array}$ \\
\hline Total numbers & & 9,766 & 691,862 & - & 9,766 & - \\
\hline Age (years) & & $60.3 \pm 15.3$ & $47.8 \pm 18.7$ & 0.73 & $60.5 \pm 15.3$ & 0.014 \\
\hline Body mass index & & $31.9 \pm 7.4$ & $30.5 \pm 7.4$ & 0.19 & $31.9 \pm 74$ & 0.006 \\
\hline Female & & $6,461(66.16 \%)$ & $381,609(55.16 \%)$ & 0.23 & $6401(65.54 \%)$ & 0.013 \\
\hline \multirow[t]{3}{*}{ Race } & White & $7,252(74.26 \%)$ & $427,956(61.86 \%)$ & 0.27 & $7360(75.36 \%)$ & 0.025 \\
\hline & African Americans & $1,538(15.75 \%)$ & $115,341(16.67 \%)$ & 0.02 & $1474(15.09 \%)$ & 0.018 \\
\hline & Asian & $121(1.24 \%)$ & $17,537(2.54 \%)$ & 0.09 & $115(1.18 \%)$ & 0.006 \\
\hline \multirow[t]{9}{*}{ Comorbidities } & Hypertension & $6,403(65.56 \%)$ & $177,330(25.63 \%)$ & 0.87 & $6,475(66.3 \%)$ & 0.016 \\
\hline & Chronic lung disease & $4,292(43.95 \%)$ & $104,401(15.09 \%)$ & 0.67 & $4,262(43.64 \%)$ & 0.006 \\
\hline & Diabetes mellitus & $3,220(39.97 \%)$ & $87,055(12.58 \%)$ & 0.50 & $3,183(32.59 \%)$ & 0.008 \\
\hline & Ischemic heart diseases & $2,584(26.46 \%)$ & $52,989(7.66 \%)$ & 0.52 & $2,533(25.94 \%)$ & 0.012 \\
\hline & Heart failure & $1,521(15.57 \%)$ & $29,999(4.34 \%)$ & 0.38 & $1,455(14.90 \%)$ & 0.019 \\
\hline & Chronic kidney disease & $1,766(18.08 \%)$ & $36,962(5.34 \%)$ & 0.40 & $1,708(17.49 \%)$ & 0.015 \\
\hline & Cerebrovascular accident & $1,746(17.88 \%)$ & $32,117(4.64 \%)$ & 0.43 & $1,637(16.76 \%)$ & 0.029 \\
\hline & Nicotine dependence & $1,879(19.24 \%)$ & $51,486(7.44 \%)$ & 0.35 & $1,826(18.70 \%)$ & 0.014 \\
\hline & Alcohol-related disorders & $605(6.19 \%)$ & $17,303(2.50 \%)$ & 0.18 & $518(5.30 \%)$ & 0.038 \\
\hline
\end{tabular}


Table.2 Risk ratios comparing outcomes of axial spondyloarthritis (ax-SpA) using the unmatched cohort

\begin{tabular}{|c|c|c|c|c|}
\hline Parameter & $\begin{array}{l}\text { Ax-SpA with COVID } \\
(n=9,841)\end{array}$ & $\begin{array}{l}\text { Non-ax-SpA with COVID } \\
(n=704,456)\end{array}$ & Risk ratio $(95 \% \mathrm{CI})$ & $p$ \\
\hline Mortality & $227(2.31 \%)$ & $12065(1.71 \%)$ & $1.35(1.18,1.53)$ & $<0.0001$ \\
\hline Hospitalization & $1933(19.64 \%)$ & $103704(14.72 \%)$ & $1.33(1.28,1.39)$ & $<0.0001$ \\
\hline Acute kidney failure & $677(6.88 \%)$ & $27332(3.9 \%)$ & $1.77(1.65,1.91)$ & $<0.0001$ \\
\hline Renal replacement therapy & $53(0.55 \%)$ & $2216(0.32 \%)$ & $1.73(1.32,2.27)$ & $<0.0001$ \\
\hline Acute respiratory distress syndrome & $192(1.95 \%)$ & $8285(1.18 \%)$ & $1.66(1.44,1.91)$ & $<0.0001$ \\
\hline Critical care services & $401(4.07 \%)$ & $18006(2.56 \%)$ & $1.59(1.45,1.76)$ & $<0.0001$ \\
\hline Mechanical ventilation & $228(2.32 \%)$ & $12695(1.80 \%)$ & $1.29(1.13,1.46)$ & 0.0001 \\
\hline Severe COVID-19 (mortality + ventilation) & $356(3.62 \%)$ & $19233(2.7 \%)$ & $1.32(1.19,1.47)$ & $<0.0001$ \\
\hline Cerebral infarction & $140(1.42 \%)$ & $4497(0.64 \%)$ & $2.23(1.89,2.63)$ & $<0.0001$ \\
\hline Venous embolism and thrombosis & $320(3.25 \%)$ & $10869(1.54 \%)$ & $2.11(1.89,2.35)$ & $<0.0001$ \\
\hline Secondary sepsis & $461(4.68 \%)$ & $21590(3.1 \%)$ & $1.53(1.40,1.67)$ & $<0.0001$ \\
\hline
\end{tabular}

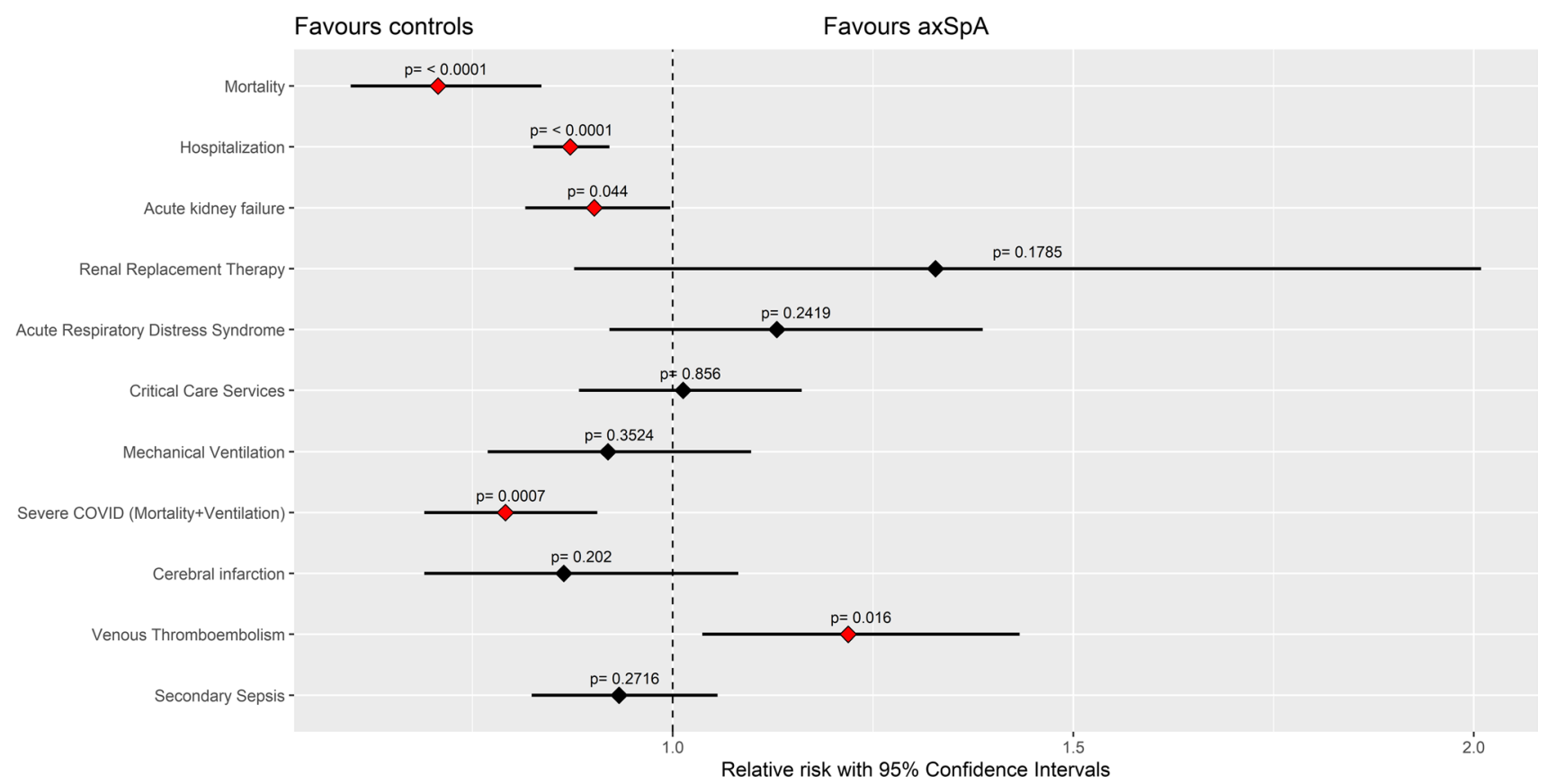

Fig. 1 Relative risk of outcomes of COVID-19 in patients with axial spondyloarthritis as compared with propensity score matched controls. Matching has been carried out for demographic characteristics (age, sex, race, body mass index) and the presence of comorbidities (hypertension, chronic lower lung disease, diabetes mellitus, ischemic heart disease, chronic kidney disease, heart failure, cerebrovascular disease, nicotine dependence, and alcohol-related disorders) exclusion of all inflammatory arthritis, 691,862 patients who had COVID-19 were identified. Demographics and prevalence of different comorbidities in ax-SpA and the others are presented in Table 1. After matching, 9766 controls were identified with a standardized difference of all matched parameters less than 0.04 (Table 1).

In the unmatched comparison, patients with ax-SpA had higher risk ratios (RR) for all 11 outcomes (Table 2). When compared with the PS matched controls (Fig. 1), patients with ax-SpA had lower RR for mortality [RR: 0.71 (95\% CI: 0.60-0.84), $p<0.0001]$, severe COVID-19 [RR: $0.79(0.69-0.91), p=0.0007]$, hospitalization [RR: 0.87 (0.83-0.92), $p<0.0001]$, and AKI [RR: 0.90 (0.82-0.997), $p=0.044]$. Only the risk of VTE was higher in ax-SpA patients [RR: 1.22 (1.04-1.43), $p=0.016]$. 


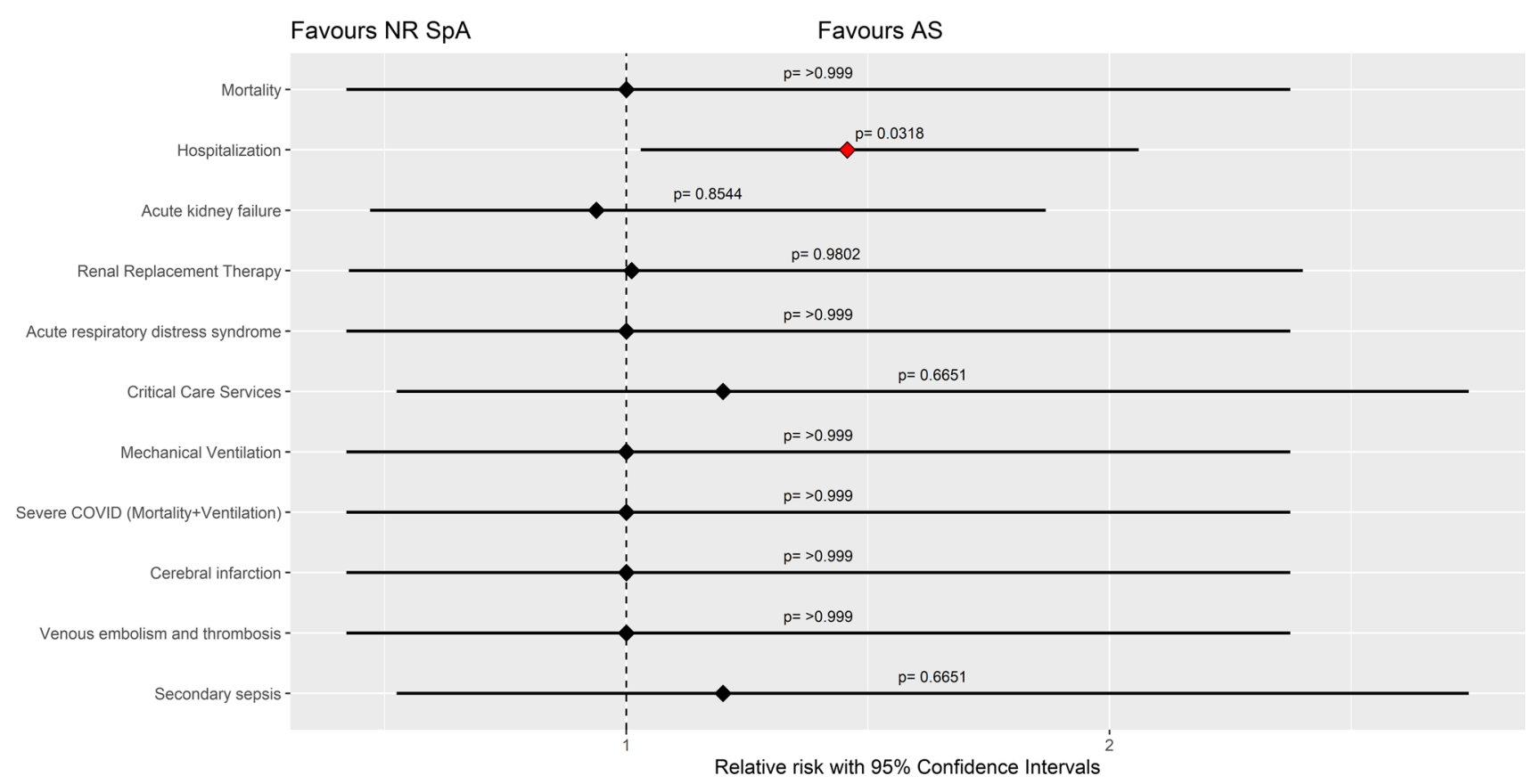

Fig. 2 Relative risk of outcomes of COVID-19 in patients with ankylosing spondylitis as compared with non-radiographic axial spondyloarthritis. PS-based matching has been carried out for demographic characteristics (age, sex, race, body mass index) and the presence of comorbidities (hypertension, chronic lower lung disease, diabetes mellitus, ischemic heart disease, chronic kidney disease, heart failure, cerebrovascular disease, nicotine dependence, and alcohol-related disorders)

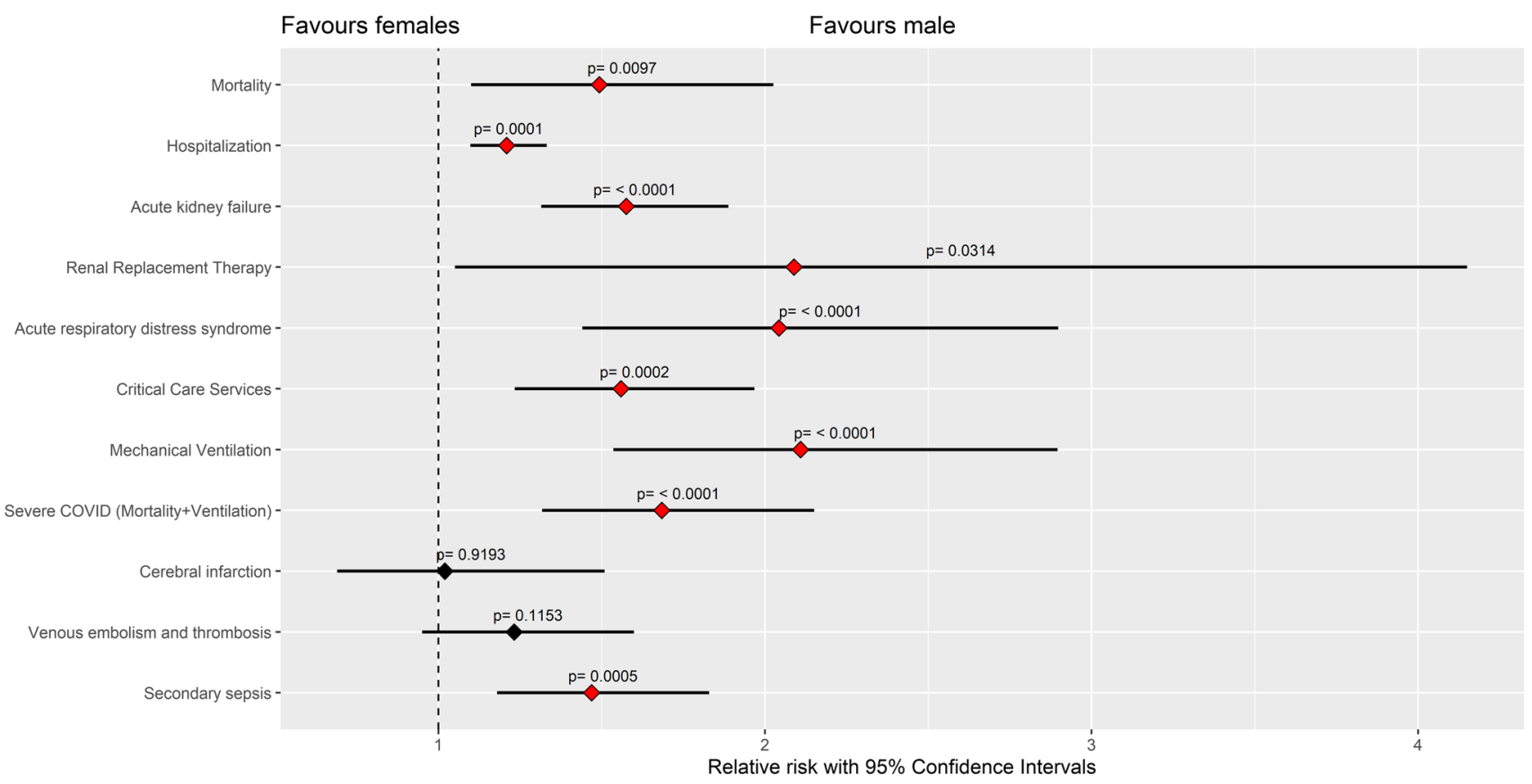

Fig. 3 Relative risk of outcomes of COVID-19 in males with axial spondyloarthritis as compared with females. PS-based matching has been carried out for demographic characteristics (age, sex, race, body mass index) and the presence of comorbidities (hypertension, chronic lower lung disease, diabetes mellitus, ischemic heart disease, chronic kidney disease, heart failure, cerebrovascular disease, nicotine dependence, and alcohol-related disorders) 


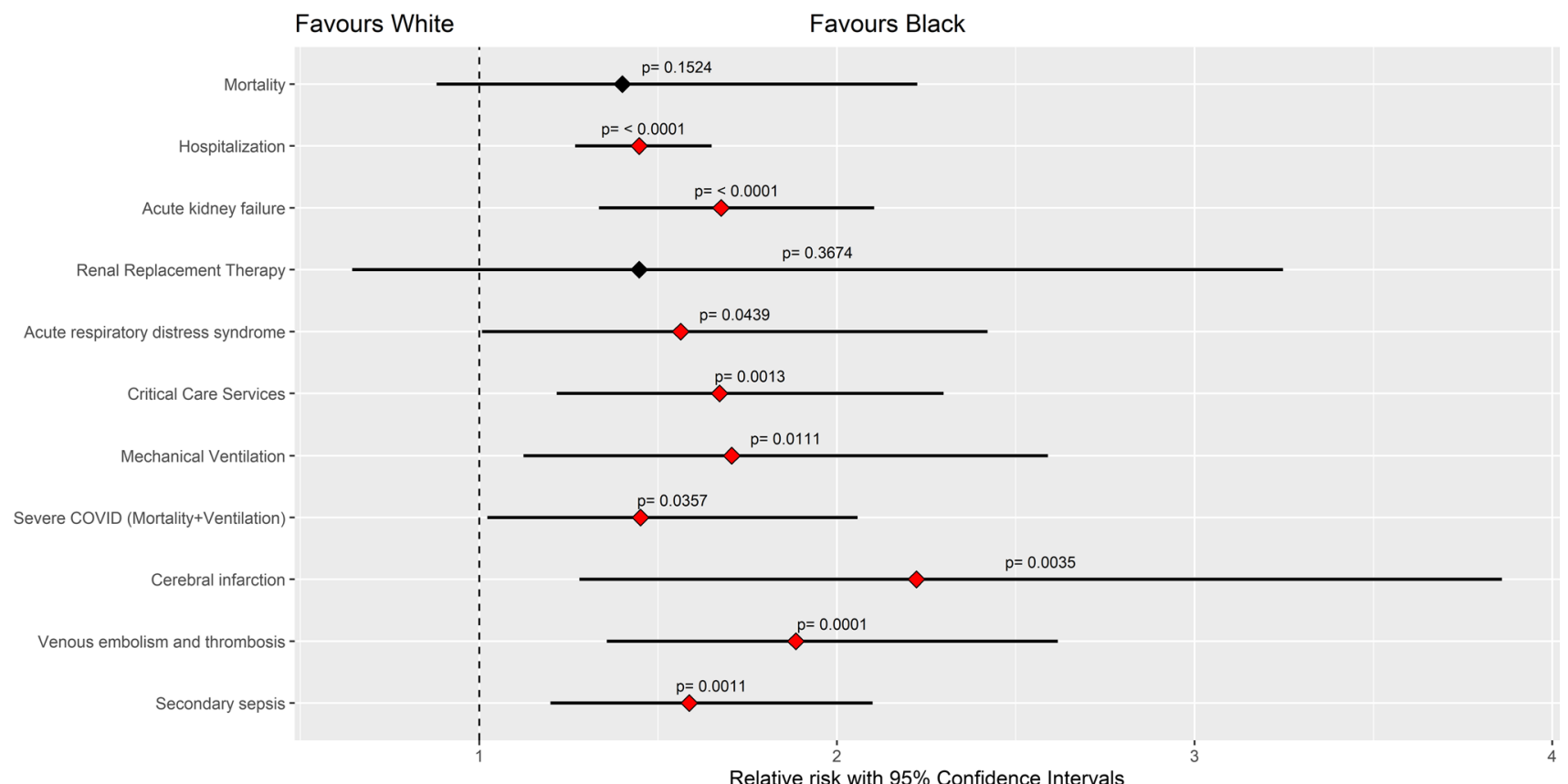

Fig. 4 Relative risk of outcomes of COVID-19 in black patients with axial spondyloarthritis as compared with white patients. PS-based matching has been carried out for demographic characteristics (age, sex, race, body mass index) and the presence of comorbidities (hyper- tension, chronic lower lung disease, diabetes mellitus, ischemic heart disease, chronic kidney disease, heart failure, cerebrovascular disease, nicotine dependence, and alcohol-related disorders)

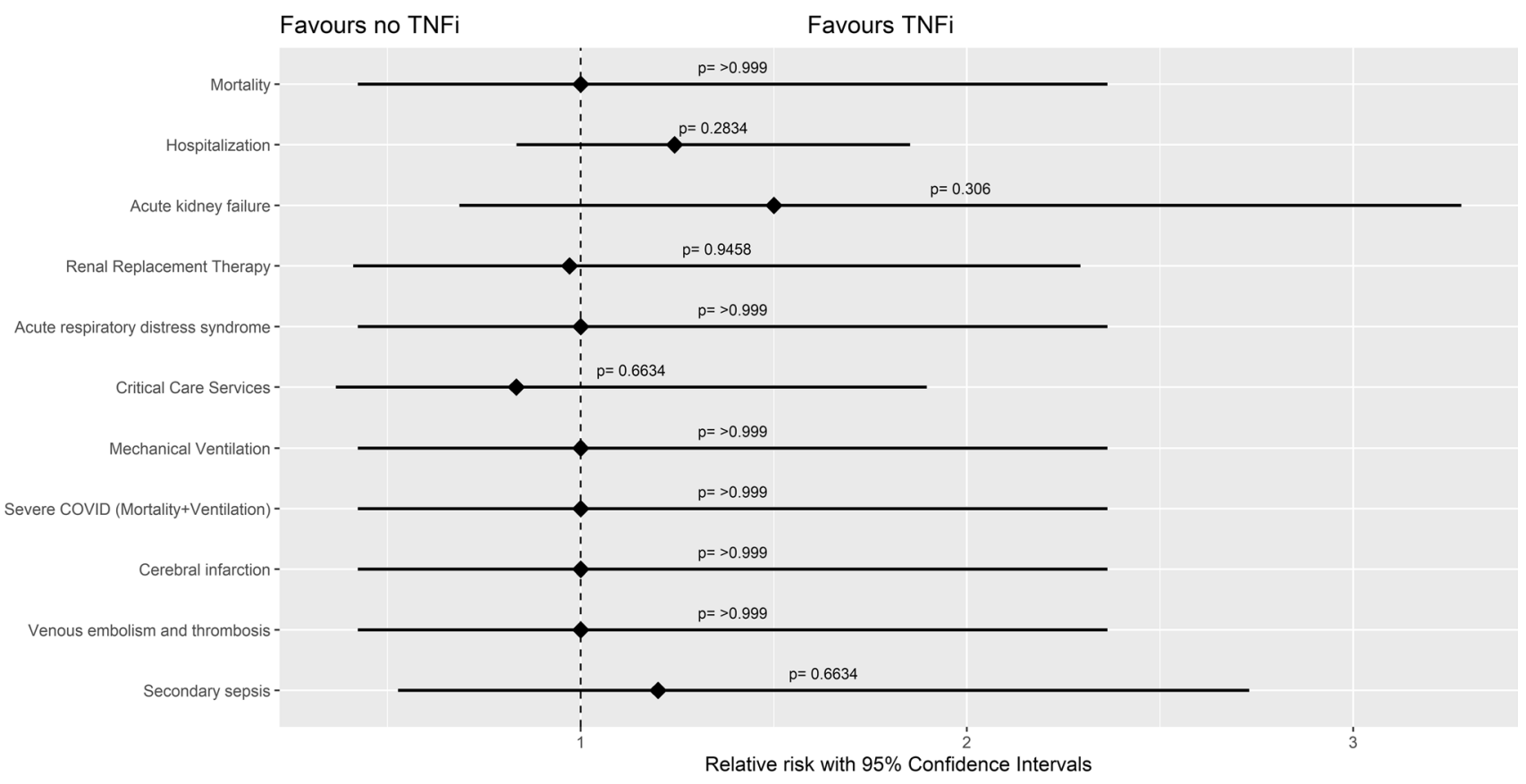

Fig. 5 Relative risk of outcomes of COVID-19 in patients with axial spondyloarthritis who received tumour necrosis factor inhibitors in previous 12 months as compared with others. PS-based matching has been carried out for demographic characteristics (age, sex, race, body mass index) and the presence of comorbidities (hypertension, chronic lower lung disease, diabetes mellitus, ischemic heart disease, chronic kidney disease, heart failure, cerebrovascular disease, nicotine dependence, and alcohol-related disorders) 


\section{Sub-group analysis}

Firstly, the difference in outcomes between AS and nr-SpA was compared. Both had a similar risk ratio except for hospitalization [RR: 1.457 (1.03-2.06), $p=0.0318$ ], which was higher in patients with AS (Fig. 2). Second, the effects of sex and race were explored. Males had worse outcomes in 9 out of the 11 domains except for VTE and cerebral infarction (Fig. 3). Blacks had worse outcomes in all domains except for mortality and the need for RRT as compared with whites (Fig. 4).

To explain the association of ax-SpA with better outcomes, we attempted to analyse the effects of TNFi. After matching for other factors, TNFi usage in the last 1 year did not significantly affect any outcome (Fig. 5). We repeated the analysis matching for TNFi use ever (data not shown), but the apparently protective effects of ax-SpA persisted in this model. The usage of interleukin-17 inhibitors was limited to 45 patients, and thus, we did not analyse the individual effects of this group of drugs. Finally, we attempted to look at how NSAID use was associated with the outcomes. Patients who had documented use of NSAIDs in the 3 months preceding the COVID-19 infection had poorer outcomes in all 11 domains (Fig. 6).

\section{Discussion}

Analysing this large cohort of 9766 patients with COVID19 and ax-SpA, we found that they had poorer outcomes as compared to the unmatched general population. The PS matched analysis revealed that patients with ax-SpA were at lower risk for mortality and morbidity including hospitalization, AKI, or severe COVID-19 (composite of mortality and mechanical ventilation). There was no difference in the rates of renal replacement therapy, ARDS, ICU care, mechanical ventilation, cerebrovascular infarction, or secondary sepsis. Also, the sub-group analysis did not reveal any difference between AS versus nr-SpA patients except for higher hospitalization rates in AS. This may be an effect of longer disease duration or more severe disease in AS (factors like age, BMI, comorbidities, and others were controlled for).

Previous analyses of COVID-19 outcomes in patients with rheumatic diseases have shown neutral effects of the presence of rheumatic disease [16]. Any increased effects are only in univariate analysis [1] and disappear when demographics and comorbidities are adjusted for [3]. One study has reported a possible association of methotrexate, rituximab, and glucocorticoid use with hospitalization [17]. But confounding due to disease activity cannot be ruled out. Our results are in line with other studies that have shown increased risk with male gender and black race [18].

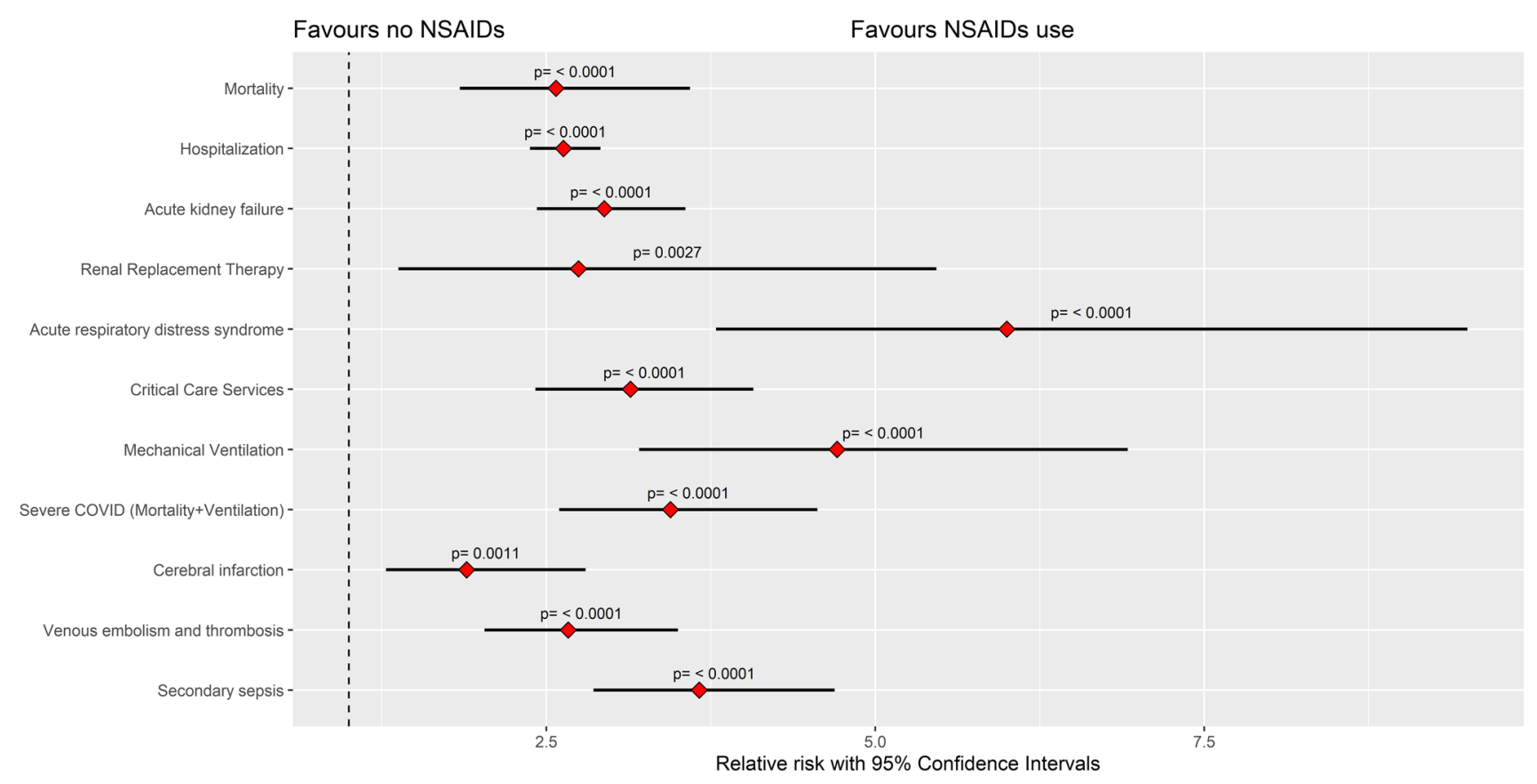

Fig. 6 Relative risk of outcomes of COVID-19 in patients with axial spondyloarthritis who were prescribed non-steroidal anti-inflammatory drugs in previous 3 months as compared with others. PS-based matching has been carried out for demographic characteristics (age, sex, race, body mass index) and the presence of comorbidities (hypertension, chronic lower lung disease, diabetes mellitus, ischemic heart disease, chronic kidney disease, heart failure, cerebrovascular disease, nicotine dependence, and alcohol-related disorders) 
An overall increased risk of VTE is expected in COVID19 [19], and this may be compounded in the presence of inflammatory arthritis that themselves increase the risk of thrombosis. Ax-SpA has been associated with an increased risk of thrombosis per se [20,21]. This may be due to genetic polymorphisms common to thrombosis and inflammatory arthritides [22]. These genes (such as SERPINE1 and IL-1 $\beta$ ) are also implicated in the pathogenesis of thrombosis in COVID-19 [19]. Similar findings of increase VTE risk during COVID-19 has also been reported for rheumatoid arthritis [23]. It can also be due to hyper viscosity, endothelial dysfunction, down-regulation of protein $\mathrm{C}$, and inhibition of fibrinolysis caused by inflammatory cytokines like TNF alpha and IL-1 [24, 25].

TNFi use within the ax-SpA patients did not reveal any reduced risks. In an analysis of an inflammatory bowel disease (IBD) database, the SECURE-IBD registry, TNFi usage was associated with a reduced risk of the composite outcome of death or hospital admission for COVID-19 (adjusted odds ratio: 0.60 [95\% CI: 0.38-0.96], $p=0.03$ ) [26]. Similarly, in a population-based study of psoriasis patients $(n=1943)$, those receiving TNFi had a lower risk of COVID-19-related hospitalization as compared with those on methotrexate (adjusted HR, 0.10; 95\% CI: 0.01-0.82) [27]. In the psoriasis cohort, none of the patients on TNFi died. In a Veterans study, TNFi use in IBD patients was not associated with an increased risk of COVID-19 infection, but the incidence was too low to analyse for severe COVID-19 [28]. In data from the COVID-19 Global Rheumatology Alliance physicianreported registry $(n=600)$, the use of TNFi (alone or in combination) was associated with a lower risk of COVID19-related hospitalization as compared with patients on no drugs (adjusted OR of 0.40 [95\% CI: $0 \cdot 19-0 \cdot 81$ ], $p=0 \cdot 01$ ) [1]. In another analysis of 77 patients with rheumatic diseases, none of the patients on TNFi $(n=16)$ needs ventilatory support or died, as opposed to $40 \%$ of patients on other therapies [29]. However, all these observational studies did not control for various possible confounders. For example, in the Global Rheumatology Alliance study [1], the comparison was with untreated patients where higher disease activity itself might be the confounding factor.

TriNetX has been previously analysed to explore the effects of TNFi usage on COVID-19 outcomes [30]. However, the number of COVID-19 cases has increased manifold since that study was carried out (total patients in the cohort was 231), and the study design was heterogeneous. Firstly, it clubbed TNFi and methotrexate together during the analysis. Secondly, it clubbed together all diseases that might be a confounding factor in itself. A sub-group analysis looked at all "diseases of the musculoskeletal system" together. However, our study design shows much clearly that TNFi usage in patients of ax-SpA does not reduce the severity of the infection.
A previous study analysing NSAID use in COVID-19 using the OpenSAFELY platform data showed that there was no added risk of the use of NSAIDs. However, in their second analysis that included only patients with rheumatoid arthritis and osteoarthritis, who are expected to be higher users of NSAIDs, current users had a better outcome than non-users [31]. To see if such a phenomenon could explain our results, we explored the association of NSAIDs use in the last 3 months but found that they were associated with poorer outcomes. This can be explained as NSAID use can increase risks of AKI and there would be confounding by higher disease activity.

Our analyses could not pinpoint an explanation why ax$\mathrm{SpA}$ was associated with better COVID-19 outcomes. One hypothesis is that the immune activation in SpA might provide a barrier against certain viral infections. HLA (Human Leukocyte Antigen)-B27 has been reported to be associated with protection against viruses like the human immunodeficiency, hepatitis C, and Epstein Barr viruses [32]. Similar to the 8.1 ancestral haplotype that predisposes to autoimmunity but is protective against certain infections [33], there might be some underlying mechanisms that can minimize certain infection risks in patients with SpA. This may be associated with the HLA class I or ERAP genes, or even some epigenetic or functional changes in immune cells due to the presence of SpA.

The limitations of our analysis include the use of aggregate data that excludes the use of multivariate analysis. The database has minimal data on disease activity, disease duration, or other relevant information like HLA-B27 positivity, insufficient for robust analysis. Also, the possible confounding by unrecognized factors cannot be excluded [34]. We cannot exclude the possibility that some AS patients might have been coded into the system as nr-SpA. However, this should not affect the overall results.

In conclusion, COVID-19 outcomes were better in patients with ax-SpA as compared with PS matched controls except for increased risk for VTE. Amongst individuals with ax-SpA, the use of TNFi does not seem to influence outcomes, but male sex and black race may confer worse COVID outcomes. This possible overall protective effect needs to be validated and explored further.

Author contribution RR, HP, and SA designed the study. RR and HP conducted statistical analysis. SA wrote the initial draft of the manuscript. All authors reviewed the study design and contributed to data interpretation and critical revision of the manuscript. All authors approved the final version for submission.

\section{Declarations}

Declaration of interests SA has received honorarium as speaker for Pfizer (unrelated to the current study) and has no other potential con- 
flicts of interest. SK has received congress travel, accommodation, and participation fee support (12th Anatolian Rheumatology Days) from AbbVie. All other authors declare no competing interests.

\section{References}

1. Gianfrancesco M, Hyrich KL, Al-Adely S et al (2020) Characteristics associated with hospitalisation for COVID-19 in people with rheumatic disease: data from the COVID-19 Global Rheumatology Alliance physician-reported registry. Ann Rheum Dis 79:859-866. https://doi.org/10.1136/annrheumdis-2020-217871

2. Jorge A, D'Silva KM, Cohen A et al (2021) Temporal trends in severe COVID-19 outcomes in patients with rheumatic disease: a cohort study. The Lancet Rheumatology 3:e131-e137. https:// doi.org/10.1016/S2665-9913(20)30422-7

3. Ahmed S, Gasparyan AY, Zimba O (2021) Comorbidities in rheumatic diseases need special consideration during the COVID-19 pandemic. Rheumatol Int 41:243-256. https://doi.org/10.1007/ s00296-020-04764-5

4. Pablos JL, Galindo M, Carmona L et al (2020) Clinical outcomes of hospitalised patients with COVID-19 and chronic inflammatory and autoimmune rheumatic diseases: a multicentric matched cohort study. Ann Rheum Dis. Published Online First. https://doi.org/10. 1136/annrheumdis-2020-218296

5. Ferri C, Giuggioli D, Raimondo V et al (2020) COVID-19 and rheumatic autoimmune systemic diseases: report of a large Italian patients series. Clin Rheumatol. Published Online First. https://doi. org/10.1007/s10067-020-05334-7

6. Duret P-M, Sebbag E, Mallick A et al (2020) Recovery from COVID-19 in a patient with spondyloarthritis treated with TNFalpha inhibitor etanercept. Ann Rheum Dis 79:1251-1252. https:// doi.org/10.1136/annrheumdis-2020-217362

7. Coskun Benlidayi I, Kurtaran B, Tirasci E, Coronavirus disease, et al (2019) (COVID-19) in a patient with ankylosing spondylitis treated with secukinumab: a case-based review. Rheumatol Int 2020:1-10. https://doi.org/10.1007/s00296-020-04635-Z

8. Misra DP, Agarwal V, Gasparyan AY et al (2020) Rheumatologists' perspective on coronavirus disease 19 (COVID-19) and potential therapeutic targets. Clin Rheumatol 39:2055-2062. https://doi.org/ 10.1007/s10067-020-05073-9

9. RECOVERY Collaborative Group, Horby P, Lim WS et al (2020) Dexamethasone in hospitalized patients with Covid-19 - preliminary report. N Engl J Med. Published Online First. https://doi.org/10. 1056/NEJMoa2021436

10. Feldmann M, Maini RN, Woody JN et al (2020) Trials of antitumour necrosis factor therapy for COVID-19 are urgently needed. Lancet 395:1407-1409. https://doi.org/10.1016/S0140-6736(20) 30858-8

11. Robinson PC, Richards D, Tanner HL et al (2020) Accumulating evidence suggests anti-TNF therapy needs to be given trial priority in COVID-19 treatment. Lancet Rheumatol 2:e653-e655. https:// doi.org/10.1016/S2665-9913(20)30309-X

12. Robinson PC, Liew DFL, Liew JW et al (2020) The potential for repurposing anti-TNF as a Therapy for the treatment of COVID-19. Med (N Y) 1:90-102. https://doi.org/10.1016/j.medj.2020.11.005

13. Singh $\mathrm{S}$, Bilal M, Pakhchanian $\mathrm{H}$ et al (2020) Impact of obesity on outcomes of patients with coronavirus disease 2019 in the United States: a multicenter electronic health records network study. Gastroenterology 159:2221-2225.e6. https://doi.org/10.1053/j.gastro.2020. 08.028

14. D'Silva KM, Jorge A, Cohen A et al (2021) COVID-19 outcomes in patients with systemic autoimmune rheumatic diseases compared to the general population: a US multicenter, comparative cohort study. Arthritis Rheumatol 73:914-920. https://doi.org/10.1002/art.41619

15. Pakhchanian H, Raiker R, Mukherjee A et al (2021) Outcomes of COVID-19 in CKD patients: a multicenter electronic medical record cohort study. Clin J Am Soc Nephrol 16:785-786. https://doi.org/ 10.2215/CJN.13820820

16. Kastritis E, Kitas GD, Vassilopoulos D et al (2020) Systemic autoimmune diseases, anti-rheumatic therapies, COVID-19 infection risk and patient outcomes. Rheumatol Int 40:1353-1360. https://doi.org/ 10.1007/s00296-020-04629-x

17. Nuño L, Navarro MN, Bonilla G et al (2020) Clinical course, severity and mortality in a cohort of patients with COVID-19 with rheumatic diseases. Annals of the Rheumatic Diseases. Published Online First. https://doi.org/10.1136/annrheumdis-2020-218054

18. Gianfrancesco MA, Leykina LA, Izadi Z et al (2021) Association of race and ethnicity with COVID-19 outcomes in rheumatic disease: data from the COVID-19 Global Rheumatology Alliance physician registry. Arthritis Rheumatol 73:374-380. https://doi.org/10.1002/ art.41567

19. Ahmed S, Zimba O, Gasparyan AY (2020) Thrombosis in Coronavirus disease 2019 (COVID-19) through the prism of Virchow's triad. Clin Rheumatol 39:2529-2543. https://doi.org/10.1007/ s10067-020-05275-1

20. Aviña-Zubieta JA, Chan J, De Vera M et al (2019) Risk of venous thromboembolism in ankylosing spondylitis: a general populationbased study. Ann Rheum Dis 78:480-485. https://doi.org/10.1136/ annrheumdis-2018-214388

21. Ungprasert P, Srivali N, Kittanamongkolchai W (2016) Ankylosing spondylitis and risk of venous thromboembolism: a systematic review and meta-analysis. Lung India 33:642-645. https://doi.org/ 10.4103/0970-2113.192862

22. Goulielmos GN, Zervou MI (2021) Risk of venous thromboembolism in ankylosing spondylitis and rheumatoid arthritis: genetic aspects. J Rheum. Published Online First. https://doi.org/10.3899/ jrheum. 210131

23. Raiker R, DeYoung C, Pakhchanian H et al (2021) Outcomes of COVID-19 in patients with rheumatoid arthritis: a multicenter research network study in the United States. Semin Arthritis Rheum 51:1057-1066. https://doi.org/10.1016/j.semarthrit.2021.08.010

24. Saha P, Smith A (2018) TNF- $\alpha$ (tumor necrosis factor- $\alpha$ ). Arterioscler Thromb Vasc Biol 38:2542-2543. https://doi.org/10.1161/ ATVBAHA.118.311660

25. Bester J, Pretorius E (2016) Effects of IL-1 $\beta$, IL-6 and IL-8 on erythrocytes, platelets and clot viscoelasticity. Sci Rep 6:32188. https:// doi.org/10.1038/srep32188

26. Brenner EJ, Ungaro RC, Gearry RB et al (2020) Corticosteroids, but not tnf antagonists, are associated with adverse COVID-19 outcomes in patients with inflammatory bowel diseases: results from an international registry. Gastroenterology 159:481-491.e3. https:// doi.org/10.1053/j.gastro.2020.05.032

27. Kridin K, Schonmann Y, Damiani G et al (2021) Tumor necrosis factor inhibitors are associated with a decreased risk of COVID19-associated hospitalization in patients with psoriasis- A population-based cohort study. Dermatol Ther. e15003. https://doi.org/10. 1111/dth. 15003

28. Khan N, Patel D, Xie D et al (2020) Impact of anti-tumor necrosis factor and thiopurine medications on the development of COVID-19 in patients with inflammatory bowel disease: a nationwide Veterans Administration cohort study. Gastroenterology 159:1545-1546.e1. https://doi.org/10.1053/j.gastro.2020.05.065

29. Winthrop KL, Brunton AE, Beekmann S et al (2021) SARS CoV-2 infection among patients using immunomodulatory therapies. Ann Rheum Dis 80:269-271. https://doi.org/10.1136/annrh eumdis-2020-218580

30. Yousaf A, Gayam S, Feldman S et al (2021) Clinical outcomes of COVID-19 in patients taking tumor necrosis factor inhibitors or 
methotrexate: a multicenter research network study. J Am Acad Dermatol 84:70-75. https://doi.org/10.1016/j.jaad.2020.09.009

31. Wong AY, MacKenna B, Morton CE et al (2021) Use of nonsteroidal anti-inflammatory drugs and risk of death from COVID19: an OpenSAFELY cohort analysis based on two cohorts. Ann Rheum Dis. Published Online First. https://doi.org/10.1136/annrh eumdis-2020-219517

32. Vitulano C, Tedeschi V, Paladini F et al (2017) The interplay between HLA-B27 and ERAP1/ERAP2 aminopeptidases: from antiviral protection to spondyloarthritis. Clin Exp Immunol 190:281290. https://doi.org/10.1111/cei.13020
33 Gambino CM, Aiello A, Accardi G et al (2018) Autoimmune diseases and 8.1 ancestral haplotype: an update. HLA 92:137-43. https://doi.org/10.1111/tan.13305

34. Ahmed S (2019) Metering the METEOR in methotrexate failure: is propensity score a falling star? Ann Rheum Dis 78:e131-e131. https://doi.org/10.1136/annrheumdis-2018-214612

Publisher's Note Springer Nature remains neutral with regard to jurisdictional claims in published maps and institutional affiliations.

\section{Authors and Affiliations}

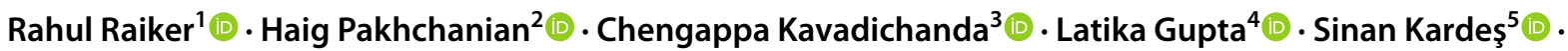 Sakir Ahmed ${ }^{6}$ (I)}

Rahul Raiker

rsraiker@gmail.com

Haig Pakhchanian

haig@gwmail.gwu.edu

Chengappa Kavadichanda

doc.chengappa@gmail.com

Latika Gupta

drlatikagupta@gmail.com

Sinan Kardeş

sinan.kardes@istanbul.edu.tr

1 West Virginia University School of Medicine, Morgantown, WV, USA

2 George Washington University School of Medicine \& Health Sciences, Washington, DC, USA
3 Department of Clinical Immunology, Jawaharlal Institute of Postgraduate Medical Education and Research (JIPMER), Puducherry, India

4 Department of Clinical Immunology and Rheumatology, Sanjay Gandhi Postgraduate Institute of Medical Sciences (SGPGI), Lucknow, Uttar Pradesh, India

5 Department of Medical Ecology and Hydroclimatology, Istanbul Faculty of Medicine, Istanbul University, Istanbul, Turkey

6 Department of Clinical Immunology and Rheumatology, Kalinga Institute of Medical Sciences (KIMS), KIIT University, Bhubaneswar, India 751024 